\title{
Evaluation of CRISPR/Cas9 site-specific function and validation of sgRNA sequence by a Cas9/sgRNA-assisted reverse PCR technique
}

\author{
Beibei Zhang ${ }^{1} \cdot$ Jiamu Zhou ${ }^{1} \cdot$ Miao Li $^{1} \cdot$ Yuanmeng Wei ${ }^{1} \cdot$ Jiaojiao Wang ${ }^{1} \cdot$ Yange Wang $^{1} \cdot$ Pingling Shi $^{1} \cdot$ Xiaoli Li $^{1}$. \\ Zixu Huang ${ }^{1} \cdot$ He Tang ${ }^{1} \cdot$ Zongming Song $^{1}$
}

Received: 9 October 2020 / Revised: 28 December 2020 / Accepted: 13 January 2021 / Published online: 4 March 2021

(C) Springer-Verlag GmbH Germany, part of Springer Nature 2021

\begin{abstract}
The effective application of the clustered regularly interspaced short palindromic repeat (CRISPR)/Cas9 system in biology, medicine and other fields is hindered by the off-target effects and loci-affinity of Cas9-sgRNA, especially at a genome-wide scale. In order to eliminate the occurrence of off-target effects and evaluate loci-affinity by CRISPR/Cas9 site-specific detection and screening of high-affinity sgRNA sequences, respectively, we develop a CRISPR/Cas9-assisted reverse PCR method for site-specific detection and sgRNA sequence validation. The detection method based on PCR can be used directly in the laboratory with PCR reaction conditions, without the need for an additional detection system, and the whole process of detection can be completed within $2 \mathrm{~h}$. Therefore, it can be easily popularized with a PCR instrument. Finally, this method is fully verified by detecting multiple forms of site mutations and evaluating the affinity of a variety of sgRNA sequences for the CRISPR/Cas9 system. In sum, it provides an effective new analysis tool for CRISPR/Cas9 genome editing-related research.
\end{abstract}

Keywords CRISPR/Cas9 $\cdot$ Site-specific detection $\cdot$ sgRNA validation

\section{Introduction}

CRISPR is widely found in the genomes of bacteria and archaea [1], as was clearly defined in 2002 [2]. It has dramatically transformed our ability to edit the genomes of various organisms, especially in eukaryotes and animal models of human disease $[3,4]$. With continuous development, in recent years CRISPR/Cas has become widely used in disciplines from basic biology to biotechnology and medicine [5-7]. Genome editing of mammalian cells was accomplished by the CRISPR/Cas9 system in 2013 [8, 9]. In 2014, genome-wide function screens in human cells were achieved $[10,11]$. While the CRISPR/Cas system has been applied mainly to the study of basic biology, in

Zongming Song

szmeye@163.com

Beibei Zhang

zhanghm126@163.com

1 Henan Provincial People's Hospital, People's Hospital of Zhengzhou University, People's Hospital of Henan University, Henan Eye Institute, Henan Eye Hospital and Henan Key Laboratory of Ophthalmology and Visual Science, Zhengzhou 450003, Henan, China
2017 Gootenberg et al. successfully engineered the system for innovative applications in nucleic acid detection [12], and numerous diverse detection techniques associated with CRISPR/ Cas systems were subsequently developed for exploring different research fields [6, 13-16]. Several researchers have explored treatments for various diseases through CRISPR/Cas systems [17-24]. However, despite the invaluable advantages of the CRISPR/Cas system (for example, easily designed and highly effective) and its wide application in biological and medical research, there are some limitations that need to be overcome.

The effective application of CRISPR/Cas systems in medicine and other fields is hindered by off-target effects, especially on a genome-wide scale $[25,26]$. When an expected target DNA is modified with the CRISPR/Cas system, unpredictable genome damage is simultaneously triggered by offtarget effects. When used in clinical trials, this can cause unanticipated risk to the health of patients. In order to eliminate or reduce the occurrence of off-target effects, several researchers have extensively explored methods for optimizing the specificity of the CRISPR/Cas system. Labun et al. designed a web tool (CHOPCHOP) for CRISPR genome engineering which is able to avoid off-target effects by identifying single-guide RNA (sgRNA) targets [27]. Even though some sgRNA selection tools contain an extensive database [28-30], 
the database is still insufficient for all genomic loci of various organisms. In addition, variations have been noted in the cleavage efficiency of the CRISPR/Cas system with different genomic loci [31], and these variances cannot be estimated with current sgRNA selection tools. In order to evaluate and validate a sitespecific sgRNA sequence, an electrochemiluminescence assay method was developed by Liu et al. [32] for detecting nucleic acid with multiple site mutations in vitro, and a label-free CRISPR/Cas9 assay method based on CuNPs was established by $\mathrm{Hu}$ et al. [33]. sgRNA sequence validation and site-specific detection can be achieved with these two methods. However, they have not achieved the popularity of PCR-based methods.

Polymerase chain reaction (PCR) was introduced in 1985 [34], and as the most common nucleic acid amplification method, it has been widely used in almost all laboratories related to biology and medicine. Although there are many novel detection methods which have certain advantages, PCR is still the most commonly used. Detection methods improved on the basis of PCR can be used directly in the laboratory with PCR reaction conditions, without an additional detection system. Therefore, PCR-based methods are easily popularized. We have developed a CRISPR- or Cas9/ sgRNA-assisted reverse PCR (CARP) method to detect and type target DNAs such as the various genotypes of the human papillomavirus [35]. CARP, derived from Cas9-sgRNA, has high specificity, so it is ideally suited for site-specific detection of CRISPR systems.

This paper demonstrates that CARP can be used for the evaluation of CRISPR/Cas9 site-specific function and screening of high-efficiency sgRNA before delivering Cas9-sgRNA vectors into cells, animal models and patients. Firstly, the single-base mutation detection ability of CARP was verified. Then we explored the effect of mutations at different sites of target DNA and sgRNA on the cleavage efficiency of Cas9sgRNA. Finally, the effects of different sgRNAs on the cleavage efficiency of Cas9 were successfully evaluated by CARP. Thus, in this study, we developed a sensitive, cost-effective and time-saving CRISPR-assisted reverse PCR method for site-specific detection and sgRNA sequence validation.

\section{Materials and methods}

\section{Preparation of sgRNA}

All oligonucleotides used in this study were synthesized by Sangon Biotechnology Co. Ltd. (Shanghai). sgRNA templates were generated in three steps (a complementary extension step and two fusion PCR amplification steps using primers listed in Table 1). Firstly, F1 and R were annealed and extended by PCR reaction for 10 cycles. A second PCR amplification step was then performed with primers $\mathrm{F} 2$ and $\mathrm{sgR}$ using the products of the previous step as template for 30 cycles. Thirdly, the last PCR reaction was performed with primers $\mathrm{F} 3$ and $\mathrm{sgR}$ using the products of the previous step as template for 30 cycles, after which the products of the third PCR were purified with a PCR purification kit. Finally, sgRNAs were synthesized by in vitro transcription. The transcription reaction was performed by incubating the purified products with RNA transcription reagents (T7 RNA polymerase, buffer and rNTPs, New England Biolabs) overnight at $37{ }^{\circ} \mathrm{C}$. The transcription products were mixed with TRIzol solution, and then successively purified with extraction of chloroform and isopropanol, and precipitated with ethanol. Purified RNA was dissolved in RNase-free $\mathrm{dd}_{2} \mathrm{O}$, quantified by spectrometry and detected by polyacrylamide gel electrophoresis. The sgRNA was synthesized by in vitro transcription according to the manufacturer's instructions.

\section{Preparation of substrate DNA}

The CDS of vascular endothelial growth factor A (VEGFA, NCBI Reference Sequence: NM_001171628.1) was synthesized and integrated into pUC57 plasmid by Sangon Biotechnology Co. Ltd. All substrate DNAs (one nonmismatched sequence and 10 mutations) were amplified with the universal primers UP1 and UP2 (Table 1), which are complementary with the pUC57 plasmid sequence. The $20 \mu \mathrm{L}$ PCR reaction system consisted of $10 \mu \mathrm{L} 2 \times$ premix Taq (Takara), $500 \mathrm{nM} \mathrm{UP1}, 500 \mathrm{nM} \mathrm{UP2}$, and $10 \mathrm{ng}$ recombinant plasmid DNA. The PCR procedure was as follows: $95{ }^{\circ} \mathrm{C}$ for $5 \mathrm{~min}$; 30 cycles of $95^{\circ} \mathrm{C}$ for $30 \mathrm{~s}, 60^{\circ} \mathrm{C}$ for $20 \mathrm{~s}$, and $72{ }^{\circ} \mathrm{C}$ for $60 \mathrm{~s}$; and $72{ }^{\circ} \mathrm{C}$ for $5 \mathrm{~min}$. The PCR products were run with $1 \%$ agarose gel and recovered with a DNA Gel Extraction Kit. The purified DNA fragments were quantified with NanoDrop (Thermo) and used as the substrate DNAs for the CARP assay.

\section{PCR-based CARP assay}

All substrate DNAs were cleaved with a pair of Cas9/sgRNA complexes. The cleavage reaction, which consisted of $1 \mu \mathrm{M}$ Cas9 Nuclease (NEB), 1× Cas9 Nuclease Reaction Buffer, $300 \mathrm{nM}$ sgRNA a (al or alm1; Table 1), and $300 \mathrm{nM}$ sgRNA b (b1 or b1m1; Table 1), was pre-incubated for assembling Cas9/ sgRNA complexes $\left(10 \mathrm{~min}\right.$ at $\left.25^{\circ} \mathrm{C}\right)$. The non-mismatched substrate DNAs were separately mixed with the pre-incubated product and cleaved at $37{ }^{\circ} \mathrm{C}$ for $5 \mathrm{~min}$. Then the cleavage product was linked with $\mathrm{T} 4$ Ligase. The ligation reaction reagents $(15 \mu \mathrm{L})$ were as follows: $5 \mathrm{U}$ T4 DNA Ligase (Thermo), $1 \times \mathrm{T} 4$ Ligase Buffer, and $5 \mu \mathrm{L}$ cleavage product. The ligation reaction was incubated at $22{ }^{\circ} \mathrm{C}$ for $10 \mathrm{~min}$. Finally, $1 \mu \mathrm{L}$ ligation product was mixed with $10 \mu \mathrm{L} 2 \times$ premix Taq (Takara), $500 \mathrm{nM} \mathrm{RP1} \mathrm{and} 500 \mathrm{nM} \mathrm{RP2}$ for a traditional PCR (PCR) reaction. The PCR procedure was as follows: $95^{\circ} \mathrm{C}$ for $5 \mathrm{~min} ; 30$ cycles of $95^{\circ} \mathrm{C}$ for $20 \mathrm{~s}, 60^{\circ} \mathrm{C}$ for $20 \mathrm{~s}$, and $72{ }^{\circ} \mathrm{C}$ for $30 \mathrm{~s}$; and $72^{\circ} \mathrm{C}$ for $5 \mathrm{~min}$. The PCR product was detected with $2 \%$ agarose gel electrophoresis. 
Table 1 Oligonucleotides used to synthesize the transcriptional template of sgRNAs and perform PCR ("F2" italicized sequence regions are the same as " $R$ " partial sequences, "F3" italicized sequences regions are the same as $\mathrm{T} 7$ promoter sequences)

\begin{tabular}{ll}
\hline Usage & Primers $\left(5^{\prime}\right.$ to $\left.3^{\prime}\right)$ \\
\hline F1 & GTTTTAGAGCTAGAAATAGCAAGTTAAAATAAGGCTAGTCCGTTATCAAC \\
TTG & AAAAAAAAGCACCGACTCGGTGCCACTTTTTCAAGTTGATAACGGACTAGCC \\
R & AAAAAAAAGCACCGACTCGGTGCCACTTTTC \\
ggR & CGATCTCATCAGGGTACTCCGTTTTAGAGCTAGAAATAGCAAG \\
a1F2 & CGATCTCATCAGGGTACTCTGTTTAGAGCTAGAAATAGCAAG \\
a1m1F2 & ATGCGGATCAAACCTCACCEGTTTTAGAGCTAGAAATAGCAAG \\
b1F2 & ATGCGGATCAAACCTCACCEGTTTAGAGCTAGAAATAGCAAG \\
b1m1F2 & TTCTAATACGACTCACTATAGCGATCTCATCAGGGTACTCCG \\
a1F3 & TTCTAATACGACTCACTATAGCGATCTCATCAGGGTACTCTG \\
a1m1F3 & TTCTAATACGACTCACTATAGATGCGGATCAAACCTCACCAG \\
b1F3 & TTCTAATACGACTCACTATAGATGCGGATCAAACCTCACCCG \\
b1m1F3 & CATGAGCGGATACATATTTG \\
UP1 & TGTTCTTTCCTCGTTATCC \\
UP2 & ACTGAGGAGTCCAACATCACC \\
RP1 & ACTCCAGGCCCTCGTCATT \\
RP2 &
\end{tabular}

\section{qPCR-based CARP assay}

The cleavage reaction, which consisted of $1 \mu \mathrm{M}$ Cas9 Nuclease (NEB), $1 \times$ Cas9 Nuclease Reaction Buffer, 300 nM sgRNA a (a1m1 to a1m5, a1 to a9; only one of these sgRNA was added in each reaction), and $300 \mathrm{nM}$ sgRNA b (b1 $1 \mathrm{~m}$ to b1m5, b1 to b9; only one of these sgRNA was added in each reaction), was pre-incubated for assembling Cas9/ sgRNA complexes $\left(10 \mathrm{~min}\right.$ at $\left.25^{\circ} \mathrm{C}\right)$, along with various negative control reactions. The substrate DNAs (gradient detection: 20, 2, 0.2, 0.02, 0.002, $0 \mathrm{ng}$; other related reactions were all $20 \mathrm{ng}$ ) were separately mixed with pre-incubated product and cleaved at $37^{\circ} \mathrm{C}$ for $5 \mathrm{~min}$. Then the cleavage product was linked with $\mathrm{T} 4$ Ligase. The ligation reaction reagents $(15 \mu \mathrm{L})$ were as follows: $5 \mathrm{U}$ T4 DNA Ligase (Thermo), $1 \times \mathrm{T} 4$ Ligase Buffer, and $5 \mu \mathrm{L}$ cleavage product. The ligation reaction was incubated at $22{ }^{\circ} \mathrm{C}$ for $10 \mathrm{~min}$. Finally, $1 \mu \mathrm{L}$ ligation product was mixed with $10 \mu \mathrm{L} 2 \times$ SYBR Green Master Mix (Yeasen), $500 \mathrm{nM} \mathrm{RP1}$ and $500 \mathrm{nM}$ RP2 for quantitative PCR (qPCR) reaction. The qPCR procedure was as follows: $95^{\circ} \mathrm{C}$ for $10 \mathrm{~min}$; 40 cycles of $95{ }^{\circ} \mathrm{C}$ for $15 \mathrm{~s}$; and $60{ }^{\circ} \mathrm{C}$ for $1 \mathrm{~min}$. The reactions were carried out on a StepOnePlus real-time PCR apparatus (ABI).

\section{Results}

\section{Schematic of CARP detection}

The CARP experimental process is schematically shown in Fig. 1. There are three main steps in CARP: (1) target DNA is specifically cut by a pair of Cas $9 / \mathrm{sgRNA}$ complexes; (2) the cleaved DNA is rapidly linked by T4 DNA ligase; (3) the ligated DNA is efficiently amplified with PCR (PCR or qPCR).

\section{Ability of CARP for single-base mutation detection}

The quality of sgRNAs is crucial in this study. sgRNA templates were generated by a complementary extension step and two fusion PCR amplification steps. In the first step, two complementary chains were annealed and extended to generate a general sgRNA frame template. In the second step, $20 \mathrm{bp}$ specific sequences complemented with target DNA were added to the general sgRNA frame template by fusion PCR. In the last step, $\mathrm{T} 7$ promoter sequences were added to the amplification products of the previous step by fusion PCR. The final PCR products were used as templates to synthesize sgRNAs. The products and sgRNAs for all three steps were respectively detected by agarose gel electrophoresis (see Supplementary Information [ESM] Fig. S1) and polyacrylamide gel electrophoresis (ESM Fig. S2). The results showed that $20 \mathrm{bp}$ specific sequences and $\mathrm{T} 7$ promoter sequences were successively added to the general sgRNA frame template, and complete sgRNA templates were successfully generated. Thus, a good foundation for the whole research was established.

After sgRNAs were generated, we explored whether single-base mutations could be discriminated using CARP. Firstly, target DNA was cut by a pair of Cas9/sgRNA complexes. Then cleavage products were linked by T4 DNA Ligase. Finally, the ligated products were detected with PCR and qPCR. If target DNA was cleaved and linked successfully by a pair of Cas9/sgRNA complexes and T4 DNA Ligase, the PCR amplification was carried out normally, and there would be a 144 bp band in the electrophoretic lane. On the contrary, 


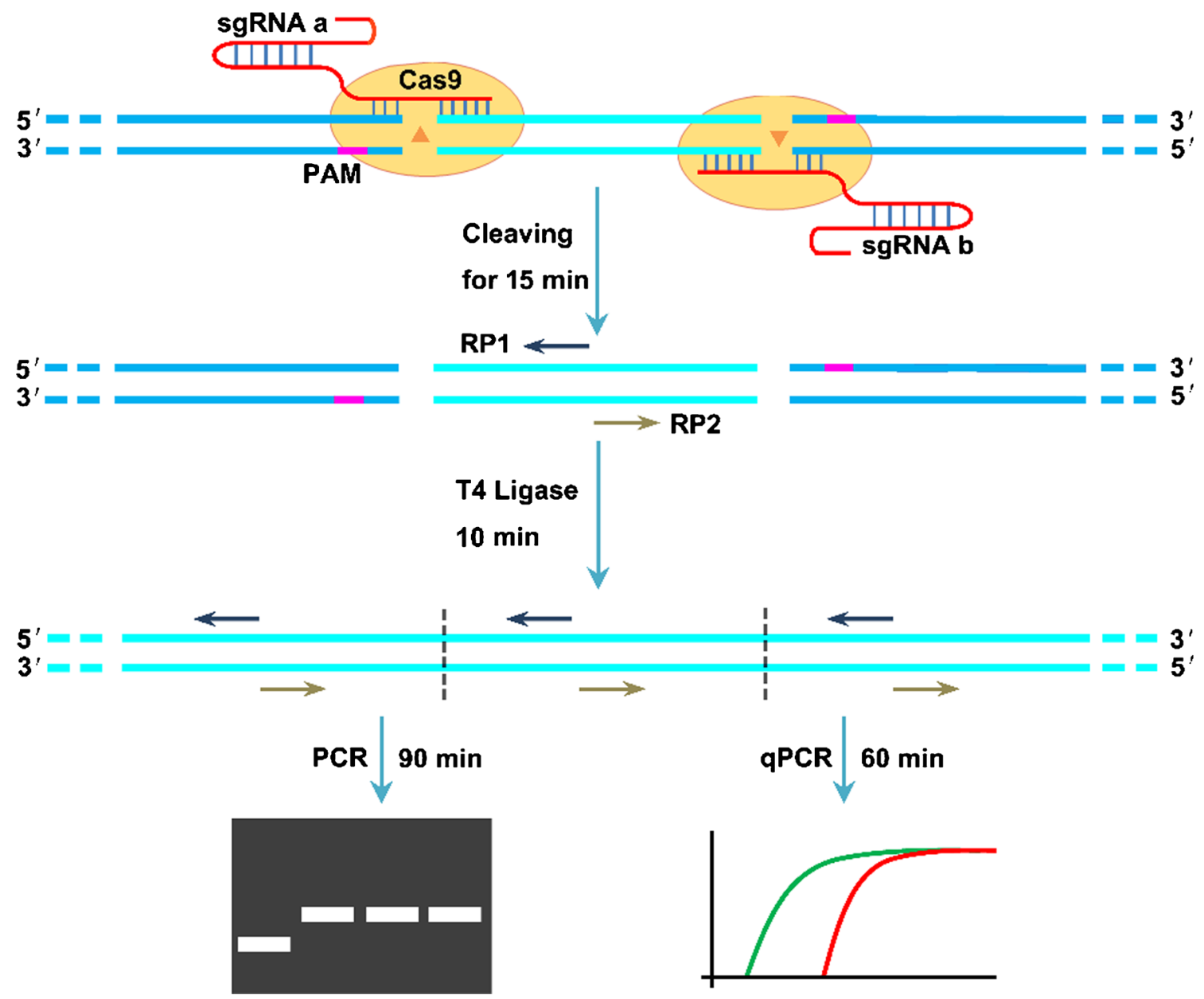

Fig. 1 Schematic illustration of CARP detection

there were no right bands in lanes of various control groups (Fig. 2a). When higher concentrations of target DNA were detected by PCR-based CARP, the bands were all in the right place (Fig. 2b). It preliminarily proved that CARP has sufficient specificity to detect the single-base mutation.

In order to further validate the specificity of CARP, the single-base mutation was detected by qPCR-based CARP (Fig. 3). Firstly, the single-base mutation was perfectly discriminated by comparing the $\mathrm{Ct}$ values of different qPCR reactions (Fig. $3 \mathrm{~b}$ and c), and there was a negative correlation between the number of ligated products and $\mathrm{Ct}$ value. Then the sensitivity of CARP was determined by detecting 20,2 , $0.2,0.02,0.002$ and $0 \mathrm{ng}$ target DNA, respectively. It was shown that as few as $0.02 \mathrm{ng}$ target DNA could be detected (Fig. 3d), compared with previous methods (limit of detection of $30 \mathrm{nM}$ and $0.13 \mathrm{nM}$ reported by Liu et al. and $\mathrm{Hu}$ et al., respectively [32,33], whereas our substrate DNA is $1766 \mathrm{bp}$, and the total volume of the reaction is $30 \mu \mathrm{L}$, so $0.02 \mathrm{ng} / 30 \mu \mathrm{L}$ can be approximately converted into $0.6 \mathrm{pM}$ ), and therefore the sensitivity of CARP is high enough to accomplish evaluation of CRISPR/Cas9 site-specific function and validation of sgRNA sequences. A standard curve of $\mathrm{Ct}$ value and a linear function with $R^{2}>0.99$ were thus generated, indicating excellent stability of the detection method (Fig. 3f). If the melting curve was used to help analyze the experimental results, the sensitivity of CARP was higher; the minimum sensitivity was $0.002 \mathrm{ng}$ (Fig. 3d). In addition, the whole detection process of CARP could be completed within $2 \mathrm{~h}$. The results fully demonstrated that CARP is able to distinguish single-base mutation. Therefore, CARP has the potential to quickly evaluate site-specific and validate sgRNA sequences.

\section{Cleavage efficiency of Cas9-sgRNA on single-base mutations of target DNA}

After determining the ability of CARP to detect single-base mutations, we investigated the site-specific of Cas9-sgRNA. First, site mutation of DNA was detected with qPCR-based CARP, and all target DNAs containing site mutations were treated with the same Cas9-sgRNA complexes. In the process of gene editing with the CRISPR/Cas system, a protospacer adjacent motif (PAM) sequence was found to be indispensable. Only DNA sequences containing PAM can be recognized and cleaved by Cas9-sgRNA. Because target DNA 

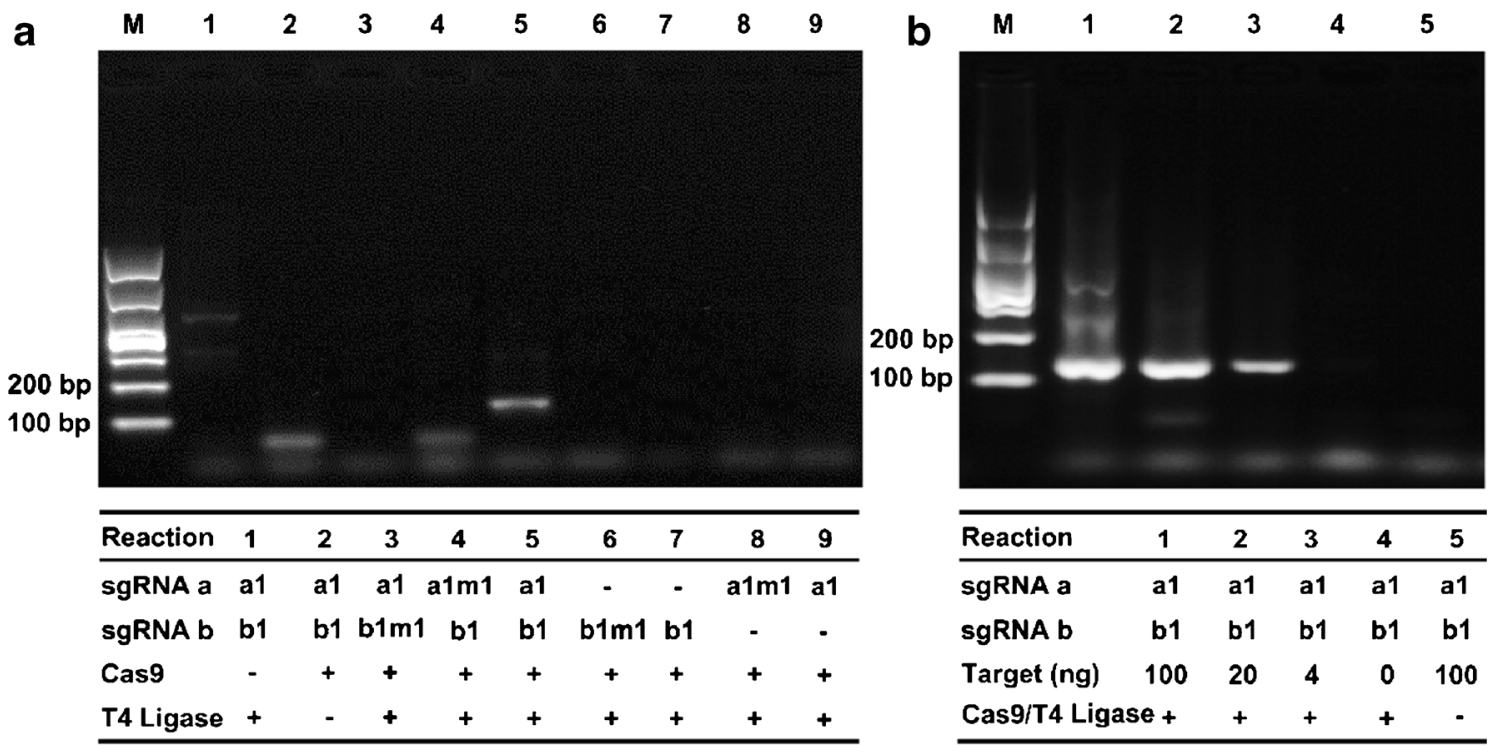

Fig. 2 CARP detection of single-base mutation (PCR). a Determining the ability of CARP to detect single-base mutation. $\mathbf{b}$ Detection of single-base mutation DNA in different concentrations. Upper figure, the experimental results; lower figure, the reaction conditions

was cleaved by Cas9 at three bases upstream of the PAM, the single-base change in PAM and its adjacent sequences were

a

\begin{tabular}{lcccccccc}
\hline Reaction & 1 & 2 & 3 & 4 & 5 & 6 & 7 & 8 \\
\hline sgRNA a & a1 & a1 & a1m & - & - & a1m & a1 & a1 \\
sgRNA b & b1 & - & - & b1 & b1m & b1 & b1m & b1 \\
Cas9 & + & + & + & + & + & + & + & + \\
T4 Ligase + & + & + & + & + & + & + & + & - \\
\hline
\end{tabular}

C

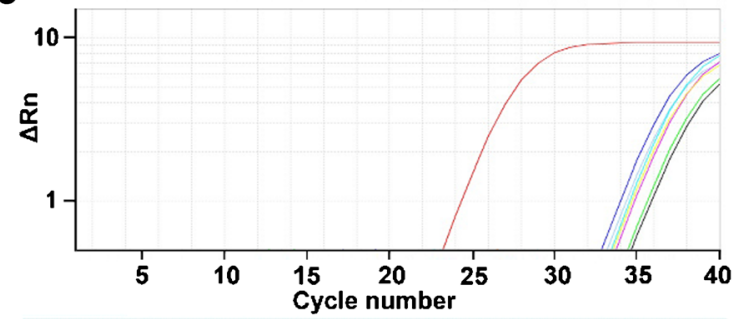

Reaction
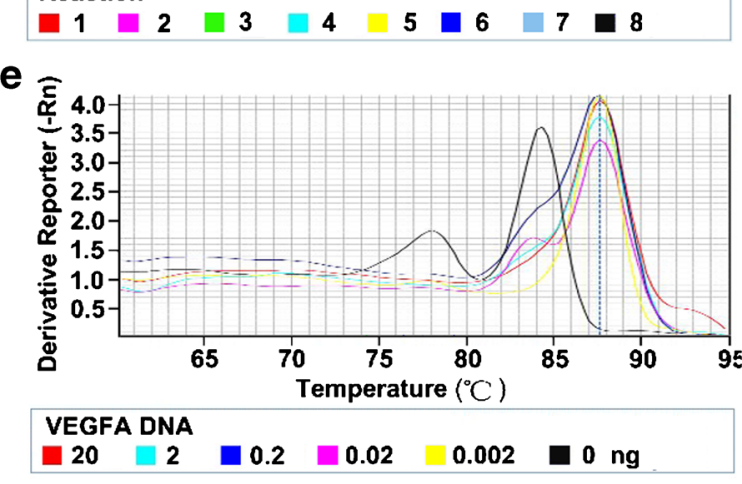

Fig. 3 CARP detection of single-base mutation (qPCR) (the error bars represent technical replicates and show standard deviation). a Detailed reaction conditions. b Quantitative detection results of single-base mutation of substrate DNA. c Logarithmic curves of qPCR for detecting explored. Five mutational strands with different single mismatch sites were designed and synthesized; the mismatch sites
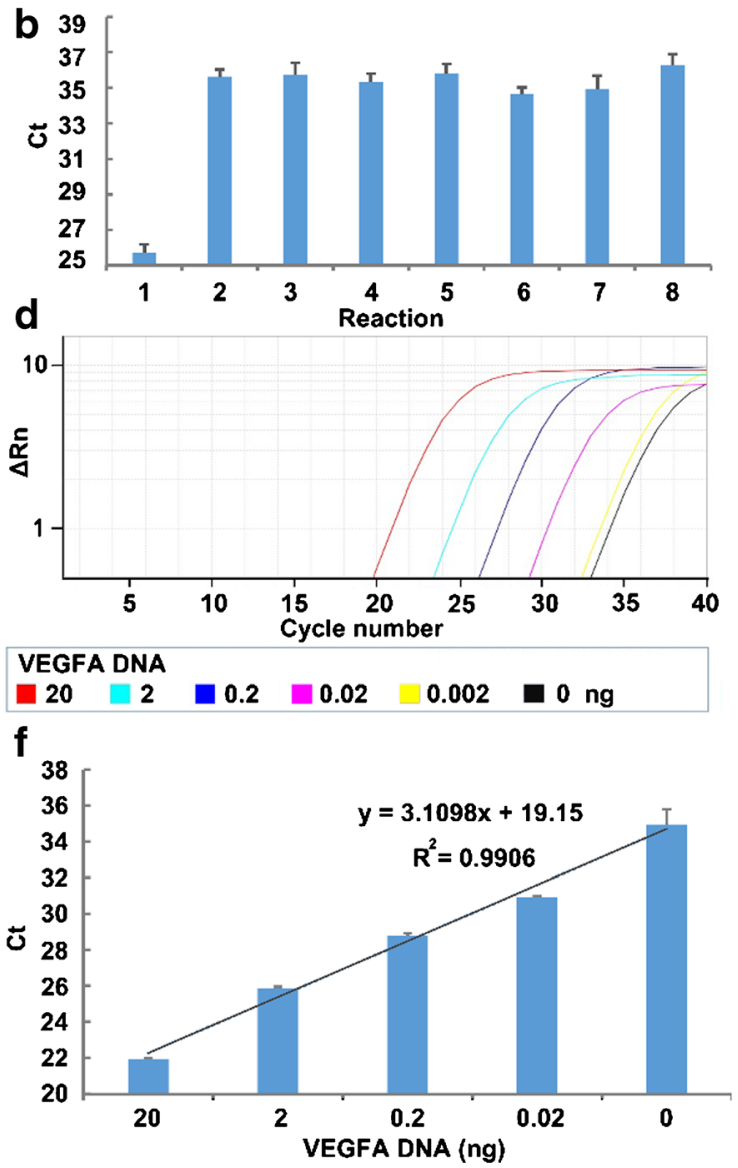

single-base mutation. d Exploring the sensitivity of detection of singlebase mutation. e The melting curves of sensitivity of CARP assay. $\mathbf{f}$ Quantitative results of the sensitivity of CARP assay 
contained three bases of the PAM and its adjacent two bases (Fig. 4a). The results show that the adjacent base mutation of PAM has little effect on cleavage efficiency of Cas9-sgRNA. Once the mutation site is in the PAM sequence, the cleavage efficiency of Cas9-sgRNA is significantly reduced. This proves that the complete PAM sequence is critical to excellent cleavage activity and affinity of the CRISPR/Cas9 system.

Spontaneous or chemical-inducible single-base mutations are common phenomena in genomic regions. Thus it is important to determine whether the cleavage activity of the CRISPR/Cas9 system can be affected by these mutations. We chose five important mutations for investigation: methylated cytosine [36, 37], two U-G base mutations (the mutation can be caused by intracellular folate deficiency) [38], and two I-C base mutations (caused by nitrite stimulation of cells) [39]. The results demonstrated that these mutations have little influence on the cleavage efficiency of the CRISPR/Cas9 system (Fig. 4b). Therefore, the effects of these mutations can be excluded in the design of Cas9-sgRNA.

\section{The effects of single-base mismatch of sgRNA on cleavage efficiency}

The specificity of the CRISPR/Cas9 system is largely determined by sgRNA which contains recognition sequences of target DNA. sgRNAs are then designed to verify whether off-target DNA can be successfully distinguished by CARP. In order to ensure the reliability of the study, we respectively designed two groups of sgRNAs based on two different sites, with each group containing five single-base mismatch sgRNAs and mismatch sites adjacent to PAM. The results revealed that the cleavage activity of the CRISPR/sgRNA system was greatly affected by a single-base mismatch of sgRNA. A small fraction of target DNA was still cut (Fig. 5), indicating that nonspecific cleavage could occur at undesigned sites. Therefore, CARP can be used to effectively avoid off-target effects before CRISPR/Cas9-related genome engineering events by exploring unpredictable DNA cleavage sites with different off-target substrate designs.
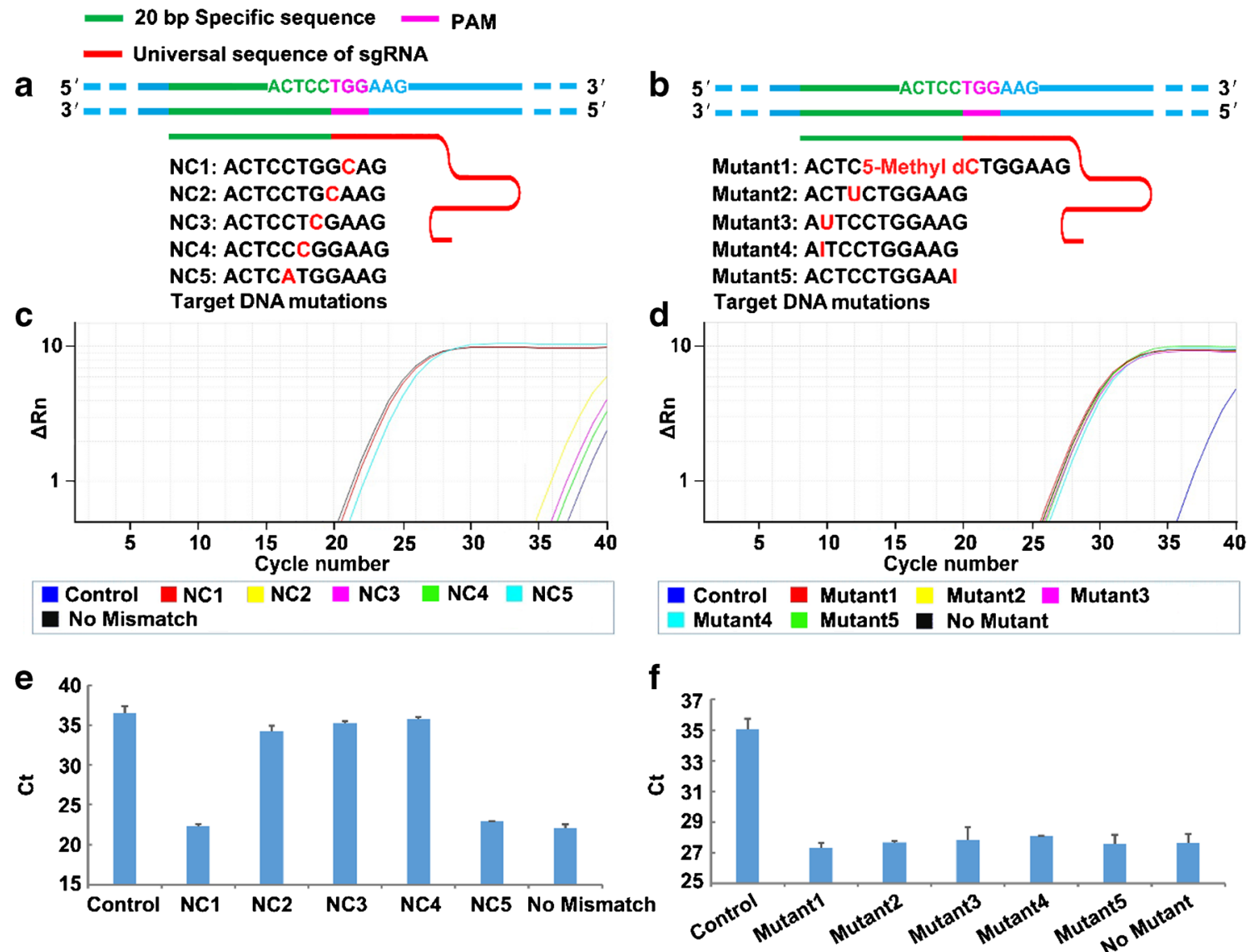

Fig. 4 Detecting single-nucleotide specificity of Cas9-sgRNA with qPCR-based CARP. a Detailed location of common mutations on substrate DNA. b Detailed location of spontaneous or chemical-inducible mutations on substrate DNA. c Detecting the single-base mismatch sequences of target DNA; non-mismatched sgRNA a1 and b1 were used in this reaction. d Detecting spontaneous or chemical-inducible single-base mutations in target DNA; non-mismatched sgRNA a1 and b1 were used in this reaction. e Quantitative detection results of common mutations of substrate DNA. f Quantitative detection results of spontaneous or chemical-inducible mutations of substrate DNA 

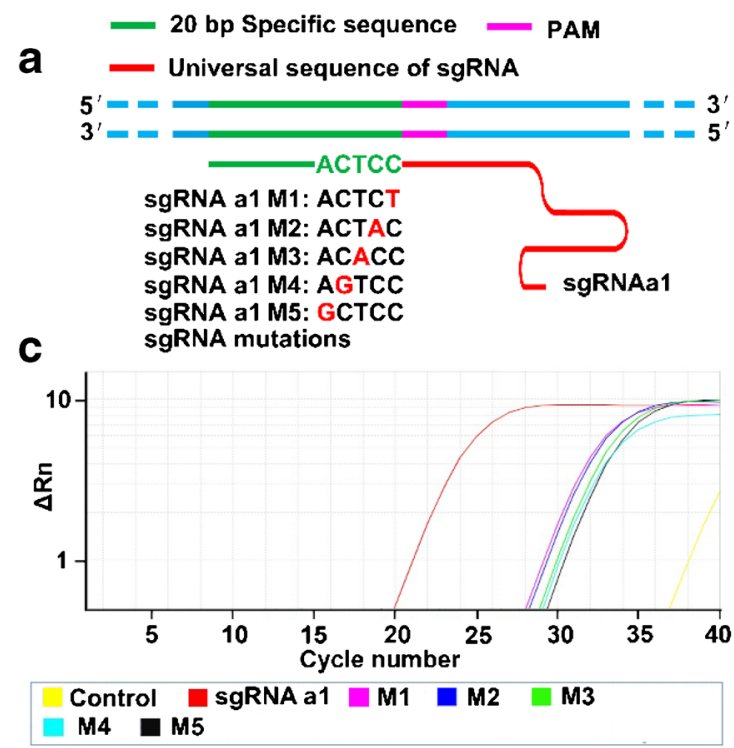

e

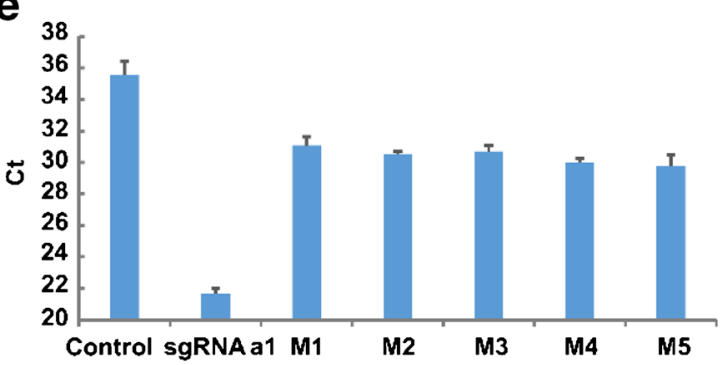

Fig. 5 Evaluating single-nucleotide specificity of sgRNA with qPCRbased CARP. a Detailed location of single-base mismatch on sgRNA a1. b Detailed location of single-base mismatch on sgRNA b1. c Evaluating the single-nucleotide specificity of sgRNA a, nonmismatched sgRNA b1 combined with one of various sgRNA al was

\section{The effects of different sgRNAs on cleavage efficiency}

Before delivering Cas9-sgRNA vectors into cells, animal models and patients, it is necessary to evaluate the affinity of sgRNA in vitro to exclude inefficient sgRNA and ensure the highest efficiency of gene editing. Finally, multiple sgRNAs (the corresponding positions of sgRNAs on target DNA, ESM Fig. S3) were designed and synthesized to detect the effects of different sgRNAs on cleavage efficiency (Fig. 6). The optimal and ineffective sgRNAs for cleavage efficiency can be successfully differentiated by comparing $\mathrm{Ct}$ values. The results suggest that the CARP method has excellent ability to screen sgRNAs with the highest affinity for CRISPR/Cas9-related gene editing.

\section{Discussion}

The CRISPR/Cas system is generally one of three major types: types I, II and III [40]. Compared with types I and III, the type II system only contains a single guided RNA b
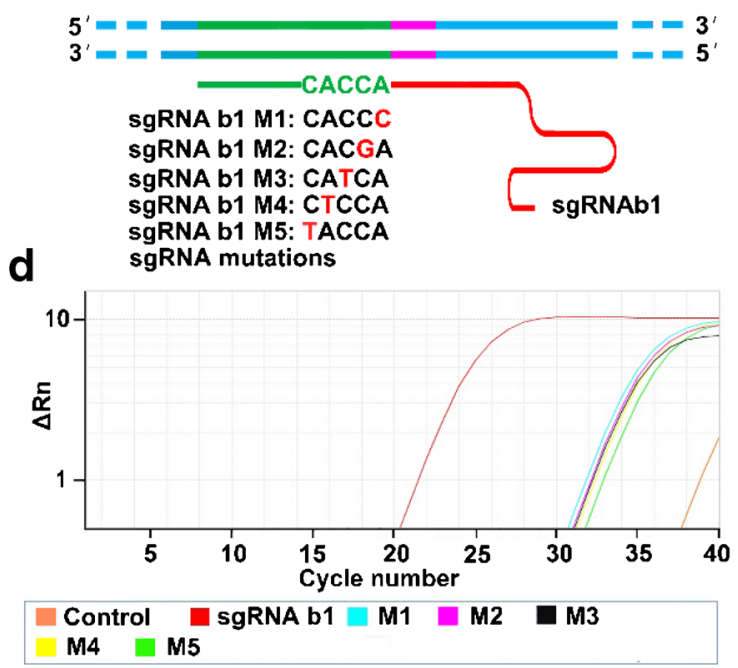

f

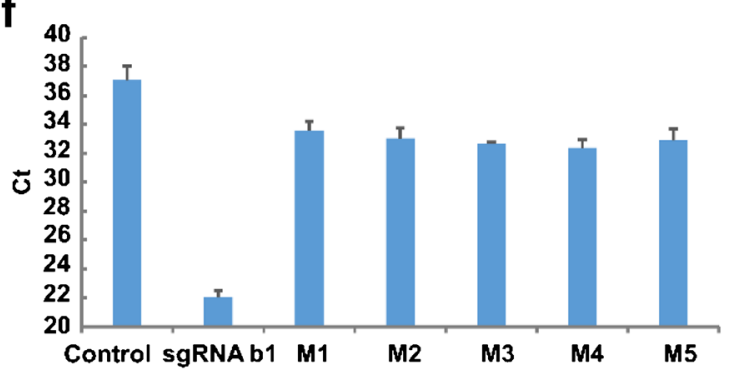

used in this reaction. d Evaluating the single-nucleotide specificity of sgRNA b, non-mismatched sgRNA a1 combined with one of various sgRNA b1 was used in this reaction. e Quantitative results of sgRNA a mutations. f Quantitative results of sgRNA b mutations

( $\operatorname{sgRNA}$ ) and one protease, such as Cas9, which is the hallmark protease of a type II system guided by sgRNA to complete a double-stranded DNA (dsDNA) cleavage event. Because of the simplicity and more mature commercial applications, we chose Cas9 as the cleavage enzyme for the CARP assay. But that does not mean that other CRISPR enzymes cannot be employed in this way. Various studies have reported the use of other types of CRISPR enzymes for nucleic acid detection. In 2017, Gootenberg et al. combined Cas13a with isothermal amplification to develop a specific high-sensitivity enzymatic reporter unlocking (SHERLOCK) technique, which provides rapid nucleic acid detection with ultra-high sensitivity and specificity [12]. Subsequently, a number of CRISPR-based nucleic acid detection technologies were established, for example, a multiplexed and portable nucleic acid detection platform with Cas13, Cas12a, and Csm6 [6], a CRISPR/Cas12a-based platform for rapid and visual nucleic acid detection [41], and an ultra-sensitive and high-throughput CRISPR-powered COVID-19 diagnostic method [42]. Both these common enzymes and other 


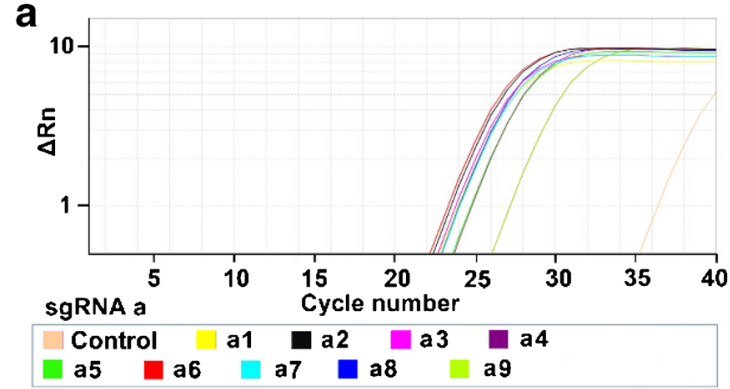

C

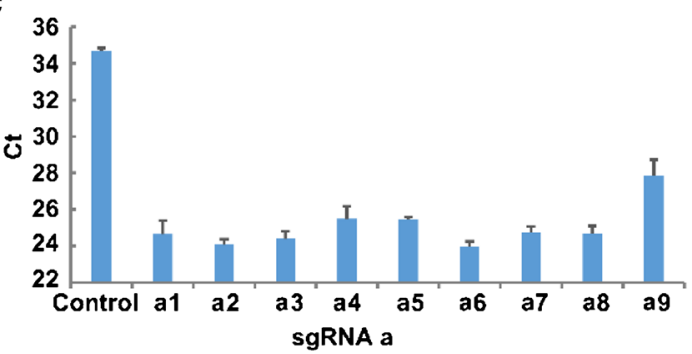

Fig. 6 Exploring the effects of different sgRNAs on cleavage efficiency simultaneously. a qPCR logarithmic curves of nine sgRNA a; one of sgRNA a1 to a9 combined with non-mismatched sgRNA b1 was used in this reaction. $\mathbf{b}$ qPCR logarithmic curves of nine sgRNA b; one of

CRISPR enzymes that have the ability to cleave DNA can be used to build an inclusive CARP detection system.

If off-target activity can be avoided, the CRISPR/Cas9 system has the ability to distinguish single-base mutations [25]. To overcome off-target activity, several approaches have been explored. A web selection tool for sgRNA, CHOPCHOP, was developed by Labun et al. to avoid off-target activity of the CRISPR/Cas9 system [27]. Double nicking (combining a Cas9 nickase with paired guide RNAs) used to enhance genome editing specificity can reduce off-target activity by 50 to 1500 -fold [43]. In this study, all sgRNA were designed with CHOPCHOP to reduce off-target activity. Double nicking was the first step of the CARP system to ensure the specificity of the detection, so that target DNA could be specifically cleaved by a pair of Cas9-sgRNA complexes. Then the cleavage products were linked by T4 DNA ligase. Finally, PCR reactions were carried out, and a pair of reverse primers was cleverly designed for amplifying target DNA. If cleavage or ligation reaction was unsuccessful, the reverse primers would elongate along the DNA template back to back, and the amplification would fail. If the target DNA was cut and the cleavage products were linked to each other into longer DNAs, the reverse primers would be changed into normal primers, and thus the target DNA was successfully amplified. On the basis of these features, the CARP system has high specificity, which was fully verified by detecting various single-base mutations.

PCR is the most common amplification method. As an exponential amplification technique, its amplification efficiency for DNA is excellent. The amplification efficiency of the CARP depends on PCR, which is the third step of the b

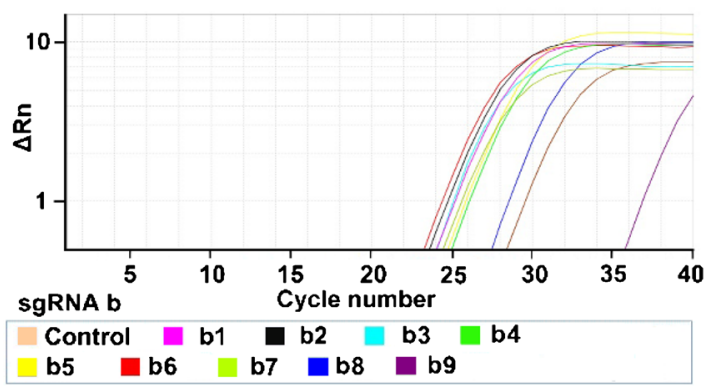

d

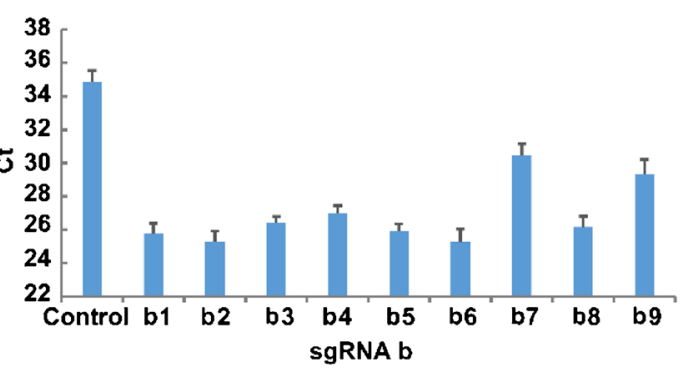

sgRNA b1 to b9 combined with non-mismatched sgRNA a1 was used in this reaction. c Quantitative results of nine sgRNA a. d Quantitative results of nine $\operatorname{sgRNA} b$

overall detection procedure, and the minimum detectable quantity in this work was $0.02 \mathrm{ng}$. Therefore, the sensitivity of our method is high enough to detect low-concentration substrate DNA. High sensitivity is a key criterion in an ideal in vitro detection method; the other key criterion is time efficiency. Compared with CRISPR/Cas9-related isothermal amplification and next-generation sequencing [44, 45], our method is a time-saving process for prescreening gene sites and sgRNA sequences. The whole CARP detection procedure can be completed within $2 \mathrm{~h}$. On the basis of the advantages above, CARP will be a promising method for evaluating sitespecific functions of CRISPR/Cas9 and validating sgRNA sequences.

\section{Conclusion}

In summary, we have developed a specific, sensitive, and time-saving Cas9/sgRNA-assisted reverse PCR method to evaluate site-specific function of CRISPR/Cas9 and validate sgRNA sequence. This method was fully verified by detecting variety site mutations and evaluating the affinity of sgRNA sequences. The detection method based on PCR can be used directly in traditional reaction conditions of PCR, without the need to establish an additional detection system. Therefore, it can be popularized easily in laboratories that have PCR instrument. According to the advantages above, it will provide a new effective analysis tool to help us explore CRISPR/Cas9 genome engineering related research. 
Supplementary Information The online version contains supplementary material available at https://doi.org/10.1007/s00216-021-03173-2.

Acknowledgments This work was supported by grants from the Key R \& D and Promotion Program of Henan Province (192102310075) and the Joint construction program of Henan medical science and technology research plan (LHGJ20200067).

\section{Declarations}

Conflict of interest The authors declare that they have no conflict of interest.

\section{References}

1. Sorek R, Kunin V, Hugenholtz P. CRISPR - a widespread system that provides acquired resistance against phages in bacteria and archaea. Nat Rev Microbiol. 2008;6(3):181-6.

2. Jansen R, van Embden JD, Gaastra W, Schouls LM. Identification of genes that are associated with DNA repeats in prokaryotes. Mol Microbiol. 2002;43(6):1565-75.

3. Mali P, Esvelt KM, Church GM. Cas9 as a versatile tool for engineering biology. Nat Methods. 2013;10(10):957-63.

4. Komor AC, Badran AH, Liu DR. CRISPR-based technologies for the manipulation of eukaryotic genomes. Cell. 2017;168(1-2):20 36.

5. Hsu PD, Lander ES, Zhang F. Development and applications of CRISPR-Cas9 for genome engineering. Cell. 2014;157(6):126278.

6. Gootenberg JS, Abudayyeh OO, Kellner MJ, Joung J, Collins JJ, Zhang F. Multiplexed and portable nucleic acid detection platform with Cas13, Cas12a, and Csm6. Science. 2018;360(6387):439-44.

7. Alagoz M, Kherad N. Advance genome editing technologies in the treatment of human diseases: CRISPR therapy. Int J Mol Med. 2020;46(2):521-34.

8. Cong L, Ran FA, Cox D, Lin S, Barretto R, Habib N, et al. Multiplex genome engineering using CRISPR/Cas systems. Science. 2013;339(6121):819-23.

9. Mali P, Yang L, Esvelt KM, Aach J, Guell M, DiCarlo JE, et al. RNA-guided human genome engineering via Cas9. Science. 2013;339(6121):823-6.

10. Wang T, Wei JJ, Sabatini DM, Lander ES. Genetic screens in human cells using the CRISPR-Cas 9 system. Science. 2014;343(6166):80-4.

11. Shalem O, Sanjana NE, Hartenian E, Shi X, Scott DA, Mikkelsen TS, et al. Genome-scale CRISPR-Cas9 knockout screening in human cells. Science. 2014;343(6166):84-7.

12. Gootenberg JS, Abudayyeh OO, Lee JW, Essletzbichler P, Dy AJ, Joung J, et al. Nucleic acid detection with CRISPR-Cas13a/C2c2. Science. 2017;356(6336):438-42.

13. Myhrvold C, Freije CA, Gootenberg JS, Abudayyeh OO, Metsky $\mathrm{HC}$, Durbin AF, et al. Field-deployable viral diagnostics using CRISPR-Cas13. Science. 2018;360(6387):444-8.

14. Hajian R, Balderston S, Tran T, DeBoer T, Etienne J, Sandhu M, et al. Detection of unamplified target genes via CRISPR-Cas9 immobilized on a graphene field-effect transistor. Nat Biomed Eng. 2019;3(6):427-37.

15. Kellner MJ, Koob JG, Gootenberg JS, Abudayyeh OO, Zhang F. SHERLOCK: nucleic acid detection with CRISPR nucleases. Nat Protoc. 2019;14(10):2986-3012.
16. Broughton JP, Deng X, Yu G, Fasching CL, Servellita V, Singh J, et al. CRISPR-Cas12-based detection of SARS-CoV-2. Nat Biotechnol. 2020;38(7):870-5.

17. Koo T, Park SW, Jo DH, Kim D, Kim JH, Cho H-Y, et al. CRISPRLbCpfl prevents choroidal neovascularization in a mouse model of age-related macular degeneration. Nat Commun. 2018;9(1):1855.

18. Maeder ML, Stefanidakis M, Wilson CJ, Baral R, Barrera LA, Bounoutas GS, et al. Development of a gene-editing approach to restore vision loss in Leber congenital amaurosis type 10. Nat Med. 2019;25(2):229-33.

19. Min Y-L, Li H, Rodriguez-Caycedo C, Mireault AA, Huang J, Shelton JM, et al. CRISPR-Cas9 corrects Duchenne muscular dystrophy exon 44 deletion mutations in mice and human cells. Sci Adv. 2019;5(3):eaav4324.

20. Li J, Wang L, Hua X, Tang H, Chen R, Yang T, et al. CRISPR/ Cas9-mediated miR-29b editing as a treatment of different types of muscle atrophy in mice. Mol Ther. 2020;28(5):1359-72.

21. Chemello F, Bassel-Duby R, Olson EN. Correction of muscular dystrophies by CRISPR gene editing. J Clin Invest. 2020;130(6): 2766-76.

22. Stadtmauer EA, Fraietta JA, Davis MM, Cohen AD, Weber KL, Lancaster E, et al. CRISPR-engineered $\mathrm{T}$ cells in patients with refractory cancer. Science. 2020;367(6481):eaba7365.

23. Dekkers JF, Whittle JR, Vaillant F, Chen H-R, Dawson C, Liu K, et al. Modeling breast cancer using CRISPR-Cas9-mediated engineering of human breast organoids. J Natl Cancer Inst. 2020;112(5): $540-4$.

24. Lu Y, Xue J, Deng T, Zhou X, Yu K, Deng L, et al. Safety and feasibility of CRISPR-edited $T$ cells in patients with refractory nonsmall-cell lung cancer. Nat Med. 2020;26(5):732-40.

25. Hsu PD, Scott DA, Weinstein JA, Ran FA, Konermann S, Agarwala V, et al. DNA targeting specificity of RNA-guided Cas9 nucleases. Nat Biotechnol. 2013;31(9):827-32.

26. Mendoza BJ, Trinh CT. Enhanced guide-RNA design and targeting analysis for precise CRISPR genome editing of single and consortia of industrially relevant and non-model organisms. Bioinformatics. 2018;34(1):16-23.

27. Labun K, Montague TG, Krause M, Torres Cleuren YN, Tjeldnes $\mathrm{H}$, Valen E. CHOPCHOP v3: expanding the CRISPR web toolbox beyond genome editing. Nucleic Acids Res. 2019;47(W1):W171W4.

28. Moreno-Mateos MA, Vejnar CE, Beaudoin J-D, Fernandez JP, Mis EK, Khokha MK, et al. CRISPRscan: designing highly efficient sgRNAs for CRISPR-Cas9 targeting in vivo. Nat Methods. 2015;12(10):982-8.

29. Ma J, Köster J, Qin Q, Hu S, Li W, Chen C, et al. CRISPR-DO for genome-wide CRISPR design and optimization. Bioinformatics. 2016;32(21):3336-8.

30. Perez AR, Pritykin Y, Vidigal JA, Chhangawala S, Zamparo L, Leslie CS, et al. GuideScan software for improved single and paired CRISPR guide RNA design. Nat Biotechnol. 2017;35(4):347-9.

31. Bassett AR, Tibbit C, Ponting CP, Liu J-L. Highly efficient targeted mutagenesis of Drosophila with the CRISPR/Cas9 system. Cell Rep. 2013;4(1):220-8.

32. Liu W, Yu H, Zhou X, Xing D. In vitro evaluation of CRISPR/Cas9 function by an electrochemiluminescent assay. Anal Chem. 2016;88(17):8369-74.

33. Hu J, Jiang M, Liu R, Lv Y. Label-free CRISPR/Cas9 assay for site-specific nucleic acid detection. Anal Chem. 2019;91(16): 10870-8.

34. Saiki RK, Scharf S, Faloona F, Mullis KB, Horn GT, Erlich HA, et al. Enzymatic amplification of beta-globin genomic sequences and restriction site analysis for diagnosis of sickle cell anemia. Science. 1985;230(4732):1350-4. 
35. Zhang B, Wang Q, Xu X, Xia Q, Long F, Li W, et al. Detection of target DNA with a novel Cas9/sgRNAs-associated reverse PCR (CARP) technique. Anal Bioanal Chem. 2018;410(12):2889-900.

36. Kumar S, Cheng X, Klimasauskas S, Mi S, Posfai J, Roberts RJ, et al. The DNA (cytosine-5) methyltransferases. Nucleic Acids Res. 1994;22(1):1-10.

37. Bestor TH, Verdine GL. DNA methyltransferases. Curr Opin Cell Biol. 1994;6(3):380-9.

38. Wickramasinghe $\mathrm{S}$, Fida $\mathrm{S}$. Bone marrow cells from vitamin B12and folate-deficient patients misincorporate uracil into DNA. Blood. 1994;83(6):1656-61.

39. Spencer JP, Whiteman M, Jenner A, Halliwell B. Nitrite-induced deamination and hypochlorite-induced oxidation of DNA in intact human respiratory tract epithelial cells. Free Radic Biol Med. 2000;28(7):1039-50.

40. Makarova KS, Haft DH, Barrangou R, Brouns SJ, Charpentier E, Horvath P, et al. Evolution and classification of the CRISPR-Cas systems. Nat Rev Microbiol. 2011;9(6):467-77.
41. Wang B, Wang R, Wang D, Wu J, Li J, Wang J, et al. Cas12aVDet: a CRISPR/Cas12a-based platform for rapid and visual nucleic acid detection. Anal Chem. 2019;91(19):12156-61.

42. Huang Z, Tian D, Liu Y, Lin Z, Lyon C, Lai W, et al. Ultrasensitive and high-throughput CRISPR-powered COVID-19 diagnosis. Biosens Bioelectron. 2020;264:112316.

43. Ran FA, Hsu PD, Lin C-Y, Gootenberg JS, Konermann S, Trevino $\mathrm{AE}$, et al. Double nicking by RNA-guided CRISPR Cas9 for enhanced genome editing specificity. Cell. 2013;154(6):1380-9.

44. Huang MQ, Zhou XM, Wang HY, Xing D. Clustered regularly interspaced short palindromic repeats/Cas9 triggered isothermal amplification for site-specific nucleic acid detection. Anal Chem. 2018;90(3):2193-200.

45. Bell CC, Magor GW, Gillinder KR, Perkins AC. A high-throughput screening strategy for detecting CRISPR-Cas9 induced mutations using next-generation sequencing. BMC Genomics. 2014;15:1002.

Publisher's note Springer Nature remains neutral with regard to jurisdictional claims in published maps and institutional affiliations. 